\title{
AVULSÃO DO PLEXO BRAQUIAL EM CÃES - 2. BIÓPSIA FASCICULAR E HISTOLOGIA DOS NERVOS RADIAL, MEDIANO, ULNAR E MUSCULO CUTÂNEO ${ }^{1}$
}

\author{
BRACHIAL PLEXUS AVULSION IN DOGS - 2. FASCICULAR BIOPSY AND HISTOLOGY \\ OF THE RADIAL, MEDIAN, ULNAR AND MUSCULOCUTANEOUS NERVES
}

\author{
Mônica Vicky Bahr Arias ${ }^{2}$ Ana Paula Frederico Loureiro Bracarense ${ }^{3}$ \\ Ângelo João Stopiglia ${ }^{4}$
}

RESUMO

O objetivo deste trabalho foi demonstrar os aspectos clínicos e neurológicos relevantes para o diagnóstico da avulsão do plexo braquial em cães, relacionando estes achados com os resultados da histologia dos nervos radiais, medianos, ulnar e músculo cutânea. A biópsia fascicular destes nervos foi realizada após abordagem cirúrgica às faces lateral e medial do braço afetado. Todos os fascículos submetidos ao exame histológico apresentaram alterações como tumefação axonal, degeneração walleriana e infiltrado inflamatório em graus variados, havendo principalmente nos nervos radial, mediano e ulnar a proliferação de colagem endoneural. A associação destes resultados com as alterações neurológicas e da eletroneuroestimulação (relatados na parte $l$ e 3 deste trabalho respectivamente) sugeriu envolvimento quase que total das raízes do plexo braquial em todos os casos.

Palavras-chave: avulsão do plexo braquial, cães, sistema nervoso periférico, biópsia.

\section{SUMMARY}

The main purposes of this work were the neurological evaluation of dogs with brachial plexus avulsion and correlation of these findings with the results of histology of the radial, median, and ulnar and muscle cutaneous nerves. Fascicular nerve biopsy was performed after surgical approach of medial and lateral aspect of the arm. Ali the submitted fascicles presented histologic alterations compatible with wallerian degeneration, axonal swelling, and inflammatory infiltrate ranging from mild to pronounced, with endoneural collagen proliferation mainly in radial, median and ulnar nerves. The association of these results with neurological and electroneurostimulation exams (respectively described in part l and 3 of this work) suggested in all cases an almost total involvement of brachial plexus roots.

Key words: brachial plexus avulsion, dogs, peripheral nervous system, and biopsy.

\section{INTRODUÇÃO}

Este estudo foi realizado devido à ocorrência relativamente comum da avulsão do plexo braquial em nossa rotina, confusão desta afeç̧ão com o quadro de paralisia do nervo radial, inexistência de trabalhos realizados de acordo com nossa realidade e principalmente, devido à dificuldade de acesso a exames complementares sofisticados.

A avulsão do plexo braquial, afecção de ordem traumática, produz a síndrome neuropática ou de neurônio motor inferior caracterizada por paralisia flácida do membro torácico com tônus muscular e reflexos segmentares ausentes ou

\footnotetext{
${ }^{1}$ Parte da tese apresentada pelo primeiro autor como requisito parcial para obtenção do título de Mestre junto ao Departamento de Cirurgia, Faculdade de Medicina Veterinária e Zootecnia (FMVZ), Universidade de São Paulo (USP). Apoio bolsa de estudos da FAPESP. ${ }^{2}$ Médico Veterinário, Professor Assistente, Departamento de Anatomia, Centro de Ciências Biológicas, Universidade Estadual de Londrina (UEL), Caixa Postal 6001, 86051-970, Londrina - PR. Autor para correspondência.

${ }^{3}$ Médico Veterinário, Professor Assistente, Departamento de Medicina Veterinária Preventiva, Centro de Ciências Agrárias, UEL.

${ }^{4}$ Médico Veterinário, Professor Associado, Departamento de Cirurgia, FMVZ, USP.
} 
diminuídos e atrofia muscular neurogênica (SHORES, 1993). A lesão pode ser incompleta, ocorrendo em alguns casos resistência de certos elementos teciduais. Alguns nervos sensoriais apresentam envolvimento parcial, havendo fibras nervosas íntegras e fibras com degeneração walleriana (GRIFFITHS, 1974).

Após compressão ou transecção nervosa, todas as fibras distais à lesão, mielinizadas ou não, sofrem degeneração walleriana, seguida ou não por um processo de regeneração (HOERLEIN, 1978). Após 24 horas ocorre desintegração da mielina e fragmentação axonal, produzindo fragmentos que serão removidos por macrófagos (HALL, 1989) e entre 12 e 36 horas após a lesão são formadas câmaras de digestão, que contém fragmentos de axônio com ovóides de mielina degenerada. A produção de mielina cessa e ocorre um aumento da população de células de Schwann formando colunas conhecidas como bandas de Büngner, que amarão como guia para os axônios do coto proximal que porventura regenerarem e como fonte de células para a mielinização destes novos axônios. Se não ocorrer a regeneração estas bandas persistem por meses, até ocorrer atrofia (CAVANAGH, 1990).

GRIFFITHS (1974), observou nos ramos ventrais dos nervos espinhais, duas a quatro semanas após o trauma, fibras degeneradas, macrófagos, proliferação das células de Schwann e fibrose intensa nos casos mais antigos. Nos nervos, as lesões eram semelhantes, embora alguns casos demonstrassem envolvimento apenas parcial, com alterações típicas de degeneração walleriana. SUMMERS et al. (1995), referem que aves selvagens com avulsão do plexo braquial, apresentavam nervos espinhais pálidos e fibróticos, havendo à microscopia óptica degeneração extensa dos axônios e formação de bandas de Büngner.

A biópsia de nervos, através da coleta de fascículos, técnica que preserva a integridade eletrofísiológica do nervo, não provoca alterações clínicas ou sensoriais, sendo procedimento útil para o diagnóstico de neuropatias. A formação de neuroma é pequena e não produz parestesia clínica durante o pós-operatório (BRAUND et al., 1979).

É importante que a biópsia não lesione vasos sanguíneos, tendões e articulações, não cause desconforto pós operatório e não predisponha o nervo à futuras lesões. Este deve ter sua localização anatômica constante e ser facilmente identificável (BRAUND et al., 1979; BRAUND, 1991; BRAUND, 1994).

GRIFFITHS

estudou

histologicamente a medula espinhal, raízes nervosas e nervos de seis cães com avulsão do plexo braquial, após estes serem submetidos à eutanásia. Em outros seis cães, estudou somente os nervos, após a realização da amputação do membro afetado. A biópsia propriamente dita é pouco utilizada rotineiramente para o diagnóstico de desordens neuromusculares em geral BRAUND et al., 1979; BRAUND, (1994) e não há descrição do seu uso em casos de avulsão do plexo braquial, pois são utilizadas outras técnicas não invasivas, como a eletromiografia (SHORES, 1993).

\section{MATERIAIS E MÉTODOS}

Para padronização da histologia e estudo anátomo-cirúrgico foram utilizados cinco cães normais, provenientes da disciplina de Técnica Cirúrgica do Departamento de Cirurgia da Faculdade de Medicina Veterinária e Zootecnia da Universidade de São Paulo (FMVZ).

Para realização da parte clínica/cirúrgica foram utilizados 12 cães de ambos os sexos, sendo oito fêmeas e quatro machos, com idade variando entre 6 meses e 11 anos, trazidos ao Hospital Veterinário da FMVZ da Universidade de São Paulo por apresentarem quadro clínico compatível com avulsão das raízes do plexo braquial. Os pacientes foram submetidos a exame clínico rotineiro, exame neurológico completo, sendo a biópsia de nervos realizada no mínimo três semanas após o traumatismo.

Para realização da biópsia fascicular, o membro afetado de cada cão foi depilado da região proximal da escápula até a parte distai do antebraço. A pré-medicação constou de clorpromazina, seguida por midazolam associado a cetamina, além de sulfato de atropina. A seguir, procedeu-se à entubação e manutenção com halotano, mantendo-se o $2^{\circ}$ plano do $3^{\circ}$ estágio anestésico de Guedel. Foi realizada a antisepsia da região tricotomizada com álcool-iodo-álcool e procedeu-se à colocação de panos de campo de modo a visualizar-se somente a região a ser operada.

Para abordagem do nervo radial foi utilizado o acesso lateral, na porção distai do úmero. A pele foi incisada paralelamente ao bordo anterior da cabeça lateral do músculo tríceps braquial, seguindo-se o mesmo plano para incisão do tecido subcutâneo, evitando-se incisar a veia cefálica. A faseia do antebraço foi incisada permitindo a visualização do nervo radial quando este ramifica-se nos ramos superficial e profundo (EVANS, 1993). A dissecção do nervo foi feita com pinça hemostática para liberá-lo dos tecidos adjacentes. Um fio de categute 6-0 foi colocado em sua borda proximal incluindo aproximadamente 1/3 da largura do nervo. Após rotação da sutura, uma tesoura oftálmica foi utilizada para dividir 
longitudinalmente o nervo. Após uma incisão transversa, os fascículos foram removidos e colocados sobre uma tira de cartolina e transferidos para solução fixadora de formol a $10 \%$. A síntese da faseia e do tecido subcutâneo foi feita com categute cromado $2-0$, e a pele suturada com pontos simples isolados com nylon $2-0$.

Para abordagem dos nervos mediano, ulnar e musculo cutâneo foi utilizado o acesso medial. A pele foi incisada paralelamente ao músculo bíceps braquial, no limite entre este e o músculo tríceps medial (EVANS, 1993), seguindose a mesma linha de incisão para os tecidos subcutâneo e adiposo. Com uma pinça de Haisted iniciou-se a divulsão para localização dos nervos e separação dos vasos (veia e artéria braquial, artéria ulnar colateral) que correm paralelamente aos nervos. Após liberação dos tecidos adjacentes, cada nervo foi identificado, utilizando-se a técnica descrita anteriormente para realização da biópsia. Do mesmo modo procedeu-se à síntese do tecido subcutâneo e pele. Após a cirurgia foi colocado penso de Robert Jones por sete dias, quando então o paciente era reavaliado procedendo-se à retirada das suturas cutâneas.

$\mathrm{O}$ fragmento de fascículo foi mantido em solução de formalina a $10 \%$ tamponada. Após a fixação, o material foi desidratado, incluído em parafina, microtomizado em cortes de $5 \mathrm{u}$ e corado com hematoxilina-eosina. $\mathrm{O}$ tecido foi então analisado em busca de alterações histológicas através de microscopia óptica de luz. Utilizou-se coloração de Tricrômico de Mallory para evidenciar presença de colágeno quando havia alterações histológicas compatíveis com proliferação de colágeno endoneural na coloração por hematoxilina eosina.

\section{RESULTADOS}

Observou-se através da coloração por hematoxilina eosina dos nervos radial, mediano, ulnar e musculo cutâneo coletado de cinco cães normais, fascículos em cortes transversais envoltos por perineuro, contendo axônios levemente basofilicos envolvidos por mielina eosinofilica reticulada. A coloração deste mesmo material pelo Tricrômico de Mallory demonstrou o axônio corado em azul claro, a mielina em vermelho e o endoneuro em azul escuro (Figura 1).

A coloração dos nervos radial, mediano, ulnar e músculo cutâneo de cães portadores de avulsão do plexo braquial pela hematoxilina eosina demonstrou alterações histológicas em todo material examinado (Figura 2 e 3). Observou-se degeneração walleriana, tumefação axonal, infiltrado inflamatório por células mononucleares intra e perivasculares, proliferação

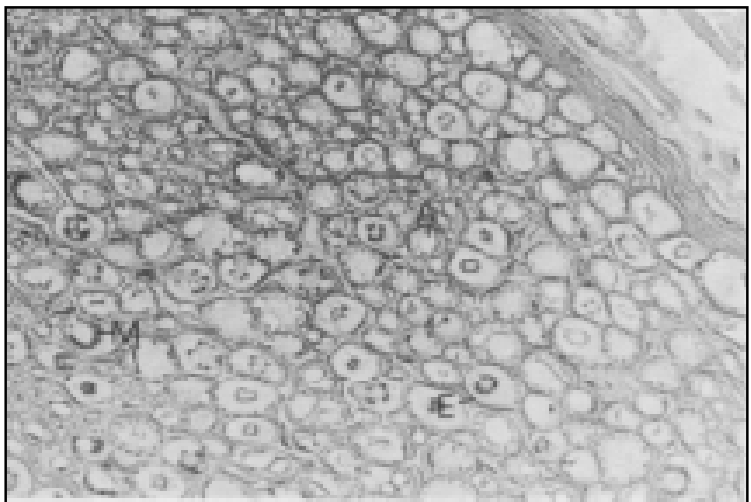

Figura 1 - Corte transversal de nervo ulnar de cão normal. Observa-se axônio (A), mielina (M) e endoneuro (E). Tricrômico de Mallory, 560X.

de fibrilas de colágeno endoneural e proliferação de células de Schwann, formando às vezes bandas de Büngner. Estas alterações foram observadas em graus leve a acentuado com presença de várias fibras nervosas íntegras. Em alguns fascículos foram observados macrófagos arredondados, de citoplasma espumoso e núcleo denso, fagocitando mielina, sendo esta alteração componente da degeneração walleriana. A coloração com Tricrômico de Mallory evidenciou a proliferação de colágeno endoneural pela presença abundante deste tecido corado em azul escuro .

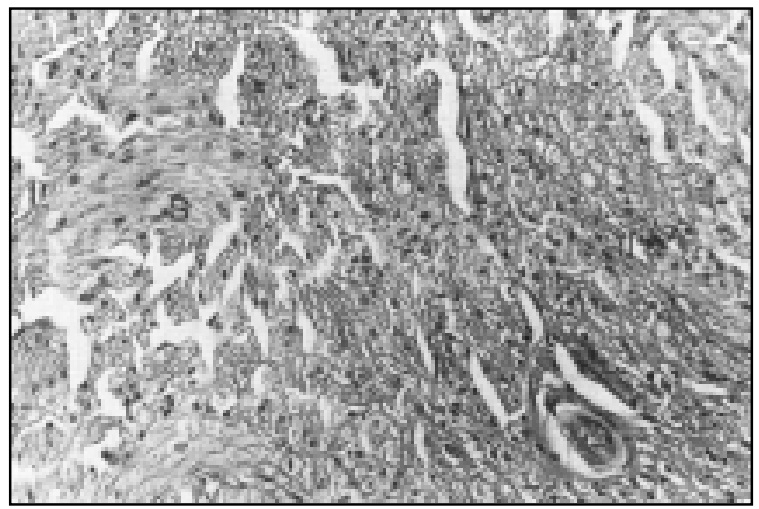

Figura 2 - Corte transversal do nervo mediano de cão com avulsão do plexo branquial. Observa-se proliferação de células de Schwann (S), tumefação axonal (t) e infiltrado inflamatório (II). Hematoxilina eosina, 440X.

Observou-se maior incidência das seguintes alterações histológicas nos nervos radial, mediano e ulnar: degeneração walleriana acentuada, infiltrado inflamatório moderado a acentuado, proliferação das células de Schwann, e estas mesmas alterações 


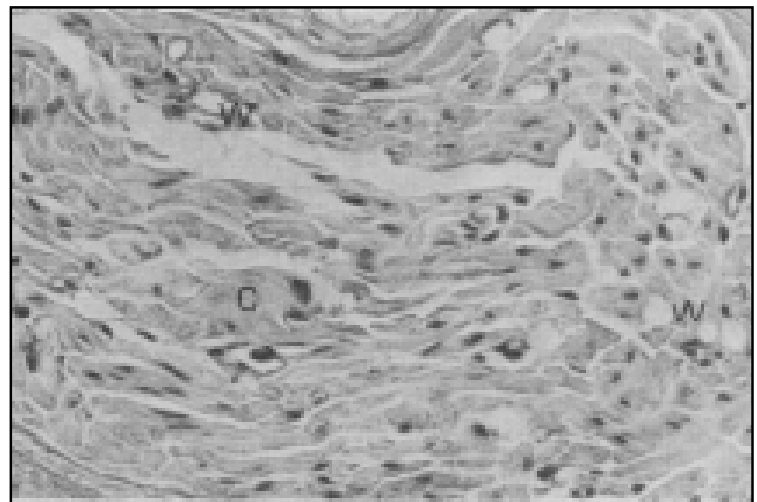

Figura 3 - Corte transversal de nervo musculocutâneo de cão com avulsão do plexo branquial. Observa-se degeneração walleriana (W) e proliferação de colágeno endoneural (C). Hematoxilina eosina, 560X.

associadas à proliferação das fibrilas de colágeno endoneural. Já a histologia do nervo musculocutâneo demonstrou maior incidência das seguintes alterações: tumefação axonal, degeneração walleriana e infiltrado inflamatório discretos a moderados (Tabela 1).

\section{DISCUSSÃO}

Tabela1 - Freqüência das alterações histológicas dso nervos radial, mediano, ulnar e n/musculocutâneo em cães portadores de avulsão do plexo branquial, trazidos ao Hospital Veterinário da Faculdade de Medicina Veterinária e Zootecnia da USP.

\begin{tabular}{|c|c|c|c|c|c|c|c|c|}
\hline \multirow{3}{*}{ Nervo } & \multicolumn{8}{|c|}{ Alteração* } \\
\hline & \multicolumn{2}{|c|}{+} & \multicolumn{2}{|c|}{++} & \multicolumn{2}{|c|}{+++} & \multicolumn{2}{|c|}{++++} \\
\hline & $\mathrm{f}$ & $\%$ & $\mathrm{f}$ & $\%$ & $\mathrm{f}$ & $\%$ & f & $\%$ \\
\hline Radial & 0 & 0 & $4 / 12$ & 33,3 & $4 / 12$ & 33,3 & $4 / 12$ & 33,3 \\
\hline Mediano & 0 & 0 & $3 / 10$ & 30,0 & $4 / 10$ & 40,0 & $3 / 10$ & 30,0 \\
\hline Ulnar & $1 / 11$ & 9,1 & $3 / 11$ & 27,3 & $3 / 11$ & 27,3 & $4 / 11$ & 36,4 \\
\hline $\begin{array}{l}\text { Muscu- } \\
\text { lo- } \\
\text { cutâneo }\end{array}$ & $2 / 10$ & 20,0 & $6 / 10$ & 60,0 & 0 & 0 & $2 / 10$ & 20,0 \\
\hline
\end{tabular}

$\mathrm{f}=$ freqüência

$*_{+}=$tumefação axonal, degeneração walleriana e inflitrado inflamatório discretos;

++ = tumafação axonal e infiltrado inflamatório moderados, degeneração walleriana discreta a moderada;

+++ = degeneração walleriana acentuada, infiltrado inflamatório moderado a acentuado, proliferação de células de Schuwann;

++++ = degeneração walleriana acentuada, infiltrado inflamatório moderado a acentuado, proliferação de células de Schuwann, proliferação das fibrilas de
Segundo SIMPSON et al. (1985) quase todos os animais com avulsão do plexo braquial apresentam envolvimento dos nervos radial, mediano e ulnar, estando os nervos musculocutâneo e axilar, frequentemente, mas nem sempre envolvidos. Entretanto, ressalta-se que no presente estudo, todos os nervos examinados apresentaram alterações histológicas em graus variados, demonstrando que raízes ou radículas que formam o nervo musculocutâneo também foram acometidas, mesmo nos casos em que houve resposta motora após a eletroneuroestimulação, achado relatado por SHORES (1993) que afirma que mesmo nas lesões caudais do plexo braquial a função deste nervo é parcial.

Segundo SWAIM (1978) a degeneração walleriana que afeia o coto distai dos nervos ocorre devido à atividade enzimática das células de Schwann, sendo os produtos desta degeneração removidos por estas mesmas células e por macrófagos. Esta atividade se inicia dois à sete dias após a lesão, alcançando um pico máximo ao redor da $3^{\text {a }}$ semana, sendo este o tempo mínimo respeitado para realização da biópsia fascicular, a qual evidenciou degeneração walleriana em graus variando de discreto à acentuado em todo material submetido à histologia.

SWAIM (1978) relata que quando não se realiza a reaproximação cirúrgica do tecido nervoso envolvido, ocorrem várias modificações nos cotos proximal e distai, principalmente a proliferação excessiva de elementos do tecido conectivo de endoneuro, perineuro e epineuro, compatível com os resultados encontrados neste estudo, onde observou-se principalmente nos nervos radial, mediano e ulnar proliferação de colágeno endoneural. Já a análise do nervo musculocutâneo evidenciou predominância de envolvimento mais discreto. GRIFFITHS (1974) e SUMMERS (1995) afirmam que devido à preservação da raiz dorsal, ou avulsão desta raiz deixar intacto o gânglio sensitivo e conseqüentemente sua projeção axonal, haveria fibras e até fascículos intactos, mesmo ocorrendo anestesia cutânea. SUMMERS (1995) ainda complementa que certas radículas podem ser destacadas, enquanto que outras pertencentes ao mesmo segmento medular podem permanecer intactas, justificando então as alterações histológicas e os resultados da eletroneuroestimulação. Estes resultados também estão de acordo com a afirmação de HOERLEIN (1978) e SIMPSON (1985) de que as raízes caudais são mais afetadas do que as craniais.

Constatou-se que a biópsia de nervo, quando utilizada conjuntamente com 
o exame neurológico e eletroneuroestimulação, forneceu resultados importantes, principalmente sobre a condição do nervo musculo cutâneo. Aparentemente, nos pacientes com capacidade de flexão do cotovelo, este nervo estaria normal, fato não observado no exame histológico. Estes resultados reforçam a opinião de BRAUND $\boldsymbol{e t} \boldsymbol{a l}$. (1979), sobre a importância da realização de exames complementares em animais com doenças neuromusculares. Observou-se entretanto, algumas dificuldades com relação à biopsia, determinantes de retirada insuficiente de material em cinco amostras. Os nervos mediano, ulnar e musculo cutâneo estão intimamente associados a vasos sanguíneos e por serem nervos cilíndricos, a separação dos fascículos é mais difícil, ao contrário do que ocorre com o nervo radial. As mesmas desvantagens foram constatadas por BRAUND (1987) na realização de biópsia fascicular do nervo ulnar.

\section{AGRADECIMENTOS}

À Professora Dra. Dominguita Ltlhers Graça, pela valiosa colaboração e sugestões na interpretação da histologia.

\section{REFERÊNCIAS BIBLIOGRÁFICAS}

BRAUND, K.G., WALTER, T.L., VANDEVELDE, M. Fascicular nerve biopsy in the dog. American Journal of Veterinary Research, v. 40,n.7,p.1025-1030, 1979.

BRAUND, K.G. Clinicai syndromes in veterinary neurology. St. Louis: Mosby, 1994. 477 p.
BRAUND, K.G. Nerve and muscle biopsy techniques. Progress in Veterinary Neurology, v. 2, n. 1, p. 35-39, 1991.

BRAUND, K.G. Diseases ofperipheral nerves, cranial nerves and muscle. In: OLIVER, J.E., HOERLEIN, B.F., MAYHEW, I.G. Veterinary neurology, Philadelphia: Saunders, 1987. p. 353-374.

CAVANAGH, F.B. Reactions of neurons and Schwann cells to injury. In: WELLER, R.O. Systemic pathology. Edinburgh: Churchill Livingstone, 1990. p. 533-543.

EVANS, H.E. Miller-s anatomy of the dog. 3 ed, Philadelphia: Saundeis, 1993. $1113 \mathrm{p}$

GR1FFITHS, I.R. Avulsion of the brachial plexus - 1 . Neuropathology of the spinal cord and peripheral nerves. Journal ofSmall Animal Practice, v. 15, p. 165-176,1974

HALL, S.M. Regeneration in the peripheral nervous system. Neuropathology and Appiied Neurobiology, v. 15, p. 513529,1989 .

HOERLEIN, B.F. Peripheral nerve distribution and fünction. In: HOERLEIN, B.F. Canine neurology. Philadelphia: Saunders, 1978.p.233-239.

SHORES, A. Traumatic and neoplastic diseases of the brachial plexus. In: BOJRAB, M.J. Diseases mechanism in fmall animais. 2. ed. Philadelphia: Lea \& Febiger, 1993. p. 1175-1182.

SIMPSON,S.T., KORNEGAY, J.N., RAFFE, M.R. Surgical diseases ofperipheral nerves. In: SLATTER, D.H. Textbook of small animal siirgery Philadelphia: Saunders, 1985. p. $1335-1338$

SUMMERS, B.A., CUMMINGS, J.F., DE LAHUNTA, A. Veterinary neuropathology. St Louis: Mosby, 1995, 527 p.

SWAIM, S.F. Peripheral nerve surgery. In. HOERLEIN, B.F Canine neurology. Philadelphia: Saunders, 1978. p.296-318. 